\title{
Influence of Complex Loading Conditions on Intervertebral Disc Failure
}

\author{
Nikolaus Berger-Roscher, Dipl.-Ing., ${ }^{*}$ Gloria Casaroli, MSEng, ${ }^{\dagger}$ Volker Rasche, PhD, ${ }^{\ddagger}, \S$ Tomaso Villa, PhD, ${ }^{\dagger,}$ \\ Fabio Galbusera, PhD, ${ }^{\top}$ and Hans-Joachim Wilke, PhD*
}

Study Design. High resolution imaging investigation of the failure of ovine lumbar intervertebral discs under complex loading.

Objective. To investigate how different loading combinations influence the mechanism and extent of intervertebral disc failure.

Summary of Background Data. Even though there has been extensive research on how an intervertebral disc fails under various conditions, failure mechanisms remain unclear. In addition, the influence of different loading directions on the mode and extent of failure under complex loading was never systematically investigated.

Methods. Thirty ovine lumbar spinal segments were loaded in a newly developed, dynamic, 6-degree-of-freedom (6-DOF) disc loading simulator under five combinations of the following loading parameters: $0^{\circ}-13^{\circ}$ flexion, $0^{\circ}-10^{\circ}$ lateral bending, $0^{\circ}-$ $4^{\circ}$ axial rotation, $0-800 \mathrm{~N}$ axial compression. A total of 1000 cycles at $2 \mathrm{~Hz}$ were done. After testing, imaging of the discs was performed in an ultra-high field magnetic resonance imaging (11.7 T) scanner and with a micro-computed tomography scanner.

Results. A total of 13 large endplate junction failures (EPJFs) occurred, of which all but one maintained an intact cartilaginous endplate. Ten out of 13 EPJFs occurred caudally. Four

From the ${ }^{*}$ Institute of Orthopedic Research and Biomechanics, Trauma Research Center Ulm (ZTF), Ulm University, Ulm, Germany; ${ }^{\dagger}$ Laboratory of Biological Structure Mechanics, Department of Chemistry, Materials and Chemical Engineering "G. Natta", Politecnico di Milano, Milan, Italy;

‡epartment of Internal Medicine II, University Hospital UIm, Ulm, Germany; ${ }^{\$}$ Small Animal MRI, Medical Faculty, Ulm University, Ulm, Germany; and "IRCCS Istituto Ortopedico Galeazzi, Milan, Italy.

Acknowledgment date: February 9, 2016. First revision date: April 13, 2016. Acceptance date: May 6, 2016.

The manuscript submitted does not contain information about medical device(s)/drug(s).

German Research Foundation (DFG) Project WI 1352/14-1 funds were received in support of this work.

Relevant financial activities outside the submitted work: grants.

Address correspondence and reprint requests to Prof. Dr. Hans-Joachim Wilke, PhD, Institute of Orthopedic Research and Biomechanics, Trauma Research Center Ulm (ZTF), Helmholtzstraße 14, 89081 Ulm, Germany; solely annulus failures occurred affecting only the outer posterior annulus. A herniation was not observed. The maximum moments measured in any group (median) were $52.5 \mathrm{~N} \cdot \mathrm{m}$ flexion, $16.5 \mathrm{~N} \cdot \mathrm{m}$ lateral bending, and $14.0 \mathrm{~N} \cdot \mathrm{m}$ axial rotation. Conclusion. Complex loading protocols could lead to EPJFs $(76 \%)$ and annulus failures $(24 \%)$ in vitro. The combination of flexion, lateral bending, axial rotation, and axial compression bears the highest risk for caudal EPJF. Flexion without lateral bending and vice versa has the lowest risk for failure. Both axial compression and axial rotation seem to have a smaller influence than flexion and lateral bending. It seems that a herniation requires an additional failure of the cartilaginous endplate, likely initiated by further axial compressive load.

Key words: annulus failure, complex loading, endplate junction failure, intervertebral disc, mechanism of herniation, micro-computed tomography, ovine lumbar motion segment, ultra-high field magnetic resonance imaging.

\section{Level of Evidence: 4}

$\mathrm{T}$ he mechanism of lumbar intervertebral disc herniation is still not fully understood. Many studies investigated the mechanical failure of the intervertebral disc with various loading protocols on human, bovine, ovine, and porcine specimens. ${ }^{1-16}$ Although all of these studies described the occurrence of herniations or protrusions, differences in set up, loading protocol, and specimens led to a lack of comparability. In addition, the techniques used for documentation of the failure morphology were limited. Thus, these studies could not explain with certainty what ultimately leads to failure of the disc.

Furthermore, focus was put on annulus rather than on endplate failure, which may also lead to disc herniation. ${ }^{17}$ Veres $e{ }^{a l} l^{18}$ were able to create defects in this area in vitro by injecting a gel into the nucleus under high pressure. Finally, Rajasekaran et $a l^{19}$ observed in 181 patients that the endplate $(65 \%)$ rather than the annulus $(35 \%)$ is subject to failure and suggested that focus in research should be put on the endplate.

Recently, Wilke et $a l^{20}$ demonstrated that it is possible to create herniations and also the damage patterns described by 
Rajasekaran et al ${ }^{19}$ in vitro with a complex loading protocol. These studies have given rise to further investigations into the failure mechanism of the intervertebral disc with focus on the endplate using a complex loading protocol. Furthermore, imaging techniques have improved, offering new insight into failure morphology.

The goal of the present study was to investigate the influence of each of four loading components-flexion $(\mathrm{FL})$, lateral bending $(\mathrm{LB})$, axial rotation (AR), axial compression $(\mathrm{AC})$ - on the initiation of intervertebral disc failure in a complex loading regime.

\section{MATERIALS AND METHODS}

Thirty lumbar spinal segments from nine healthy sheep (age $3-5$ years) were gathered. All spines were dissected and the vertebral bodies transversally bisected using a diamond band saw (Exakt, Norderstedt, Germany) to give five motion segments (L1-2, L2-3, L3-4, L4-5, L5-6). The posterior elements of the motion segments were carefully excised, providing a clear view of the posterior annulus. Screws were placed in the vertebral bodies to ensure improved fixation in the subsequent PMMA embedding (Technovit 3040, Heraeus Kulzer, Wehrheim, Germany) of the functional spinal unit (FSU). The embedded motion segments were then stored at $-20^{\circ} \mathrm{C}$. Before testing, the specimens were thawed at $6^{\circ} \mathrm{C}$ for 12 hours. Flanges were screwed onto the PMMA and mounted, unloaded, in a 6DOF dynamic spine tester ${ }^{20}$ (Figure 1).

Five groups were formed $(n=6)$; to limit the possible influence on the vertebral level, the upper and lower lumbar spines were equally represented within a group. All specimens within a group were from different spines. A compressive load of $130 \mathrm{~N}$ was applied for 15 minutes to precondition the specimens at a typical spinal load for a standing sheep. ${ }^{21}$ Testing was performed using a complex loading protocol (Table 1). The following loading parameters were used: $0^{\circ}$ to $13^{\circ} \mathrm{FL}, 0^{\circ}$ to $10^{\circ}$ right $\mathrm{LB}$, and $0^{\circ}$ to $4^{\circ}$ right AR combined with 0 to $800 \mathrm{~N}$ of AC at a frequency of $2 \mathrm{~Hz}$. The angles were chosen to be twice the values obtained by Reitmaier et $a l^{22}$ where the range of motion of ovine lumbar FSU was measured under $3.75 \mathrm{~N} \cdot \mathrm{m}$ pure moment applied in each direction without posterior elements. The frequency of $2 \mathrm{~Hz}$ with angles up to $13^{\circ}$ results in a maximum angular speed of about $82 \%$ which is deemed as physiologic for dynamic activities. ${ }^{23}$ Within the first 90 cycles, angles were increased in $10 \%$-increments of the full angle or load with 10 cycles each. A total of 1000 cycles were performed. During the test, specimens were recorded by a video camera from the posteroanterior direction to detect visible and audible disc failures. Upon test completion, the specimens were again frozen at $-20^{\circ} \mathrm{C}$. Micro-computed tomography $(\mu \mathrm{CT}$ ) (Skyscan 1172, Skyscan, Kontich, Belgium) of the frozen specimens was performed at $34 \mu \mathrm{m}$ resolution using a voltage of $100 \mathrm{kV}$ and $100 \mu \mathrm{A}$ with an acquisition time of 20 minutes. For magnetic resonance imaging (MRI) scans, the specimens were thawed at $6^{\circ} \mathrm{C}$ overnight before scanning. Imaging was performed in a $11.7 \mathrm{~T}$ small animal MRI system (BioSpec 117/16, Bruker Biospin, Ettlingen, Germany) using an experimental protocol derived from previous experiments (MRI method: FLASH, contrast: T1, echo time: $3.5 \mathrm{ms,}$ repetition time: $10.0 \mathrm{~ms}$, resolution: $100 \mu \mathrm{m}$ isotropic, slice gap: $100 \mu \mathrm{m}$, FOV: $60 \times 70 \mathrm{~mm}$, averages: 1, acquisition time: $15 \mathrm{~min}) .{ }^{24}$ All data were received with a $40 \mathrm{~mm}$ quadrature transmit/receive coil. Video documentation and scan images were visually analyzed. For image visualization, Image J (U. S. National Institutes of Health, Bethesda, MD) was used.

Analysis of load cell data was conducted using MATLAB (R2013b, The MathWorks Inc., Natick, MA). Loading
Figure 1. Schematic drawing of the test set-up. 6DOF dynamic spine tester (left) and detailed view of the mounted specimen (right), filmed from the posteroanterior direction during testing.

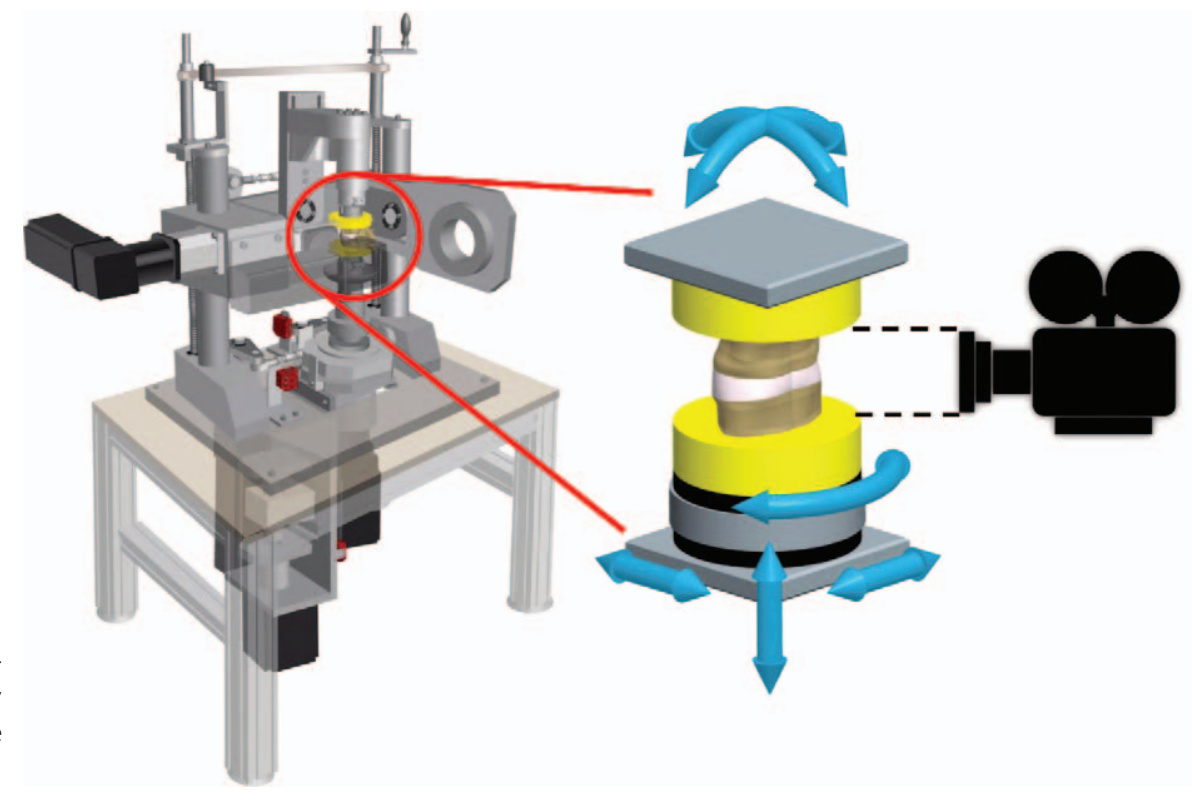




\begin{tabular}{|c|c|c|c|c|c|}
\hline Load Component & Group 1 & Group 2 & Group 3 & Group 4 & Group 5 \\
\hline FL & $x$ & $x$ & $x$ & $x$ & - \\
\hline LB & $X$ & $X$ & $X$ & - & $X$ \\
\hline AR & $X$ & $X$ & - & $X$ & $X$ \\
\hline $\mathrm{AC}$ & $X$ & - & $X$ & $X$ & $X$ \\
\hline
\end{tabular}

curves were synchronized with video documentation and analyzed with respect to audible and visible defects. Large endplate junction failures (EPJFs) were defined as bony avulsions containing both cortical and cancellous bone. Small EPJFs were defined as bony avulsions from the cortical shell only. The risk of EPJFs was calculated as large $\mathrm{EPJFs}+$ small EPJF $\times 0.5$.

\section{RESULTS}

There were a total of 17 EPJFs. Thirteen were large, 10 of which occurred at the caudal endplate (Table 2). All four small EPJFs affected both cranial and caudal endplates. Annular tears were only noticed in the outer posterior annulus. They occurred along with EPJFs in six cases and without EPJF in four cases. Site of failure was typically the median, paramedian, or left posterolateral part of the specimen. Herniations with nucleus visible outside of the specimen were not produced in any specimen.

The development of EPJFs and annular tears was audible as crackling noise in the video documentation for all cases (see Videos, Supplemental Digital Content 1, http://links. lww.com/BRS/B168 and 2, http://links.lww.com/BRS/B167 which show the posterior annulus during testing). In addition, the defects were usually easily distinguishable through visible inspection but in some cases were slightly covered by the posterior longitudinal ligament. Failure generally developed between $50 \%$ and $100 \%$ of load application.
Moments in FL were the highest of all rotational DOFs and higher with AC than without (group 1, 52.5 N.m vs. group $2,37.5 \mathrm{~N} \cdot \mathrm{m})$, whereas in group $3(32.5 \mathrm{~N} \cdot \mathrm{m})$ and group $4(30.0 \mathrm{~N} \cdot \mathrm{m})$ it did not reach the values of group 1 . Without active FL (group 5), reaction moments measured in FL were $10.0 \mathrm{~N} \cdot \mathrm{m}$. Group 4 (without LB) showed a smaller bending moment $(7.0 \mathrm{~N} \cdot \mathrm{m})$ compared with the other four groups (10.0-16.5 N.m median). AR moments were between 12.0 and $14.0 \mathrm{~N} \cdot \mathrm{m}$. Only in group 3 (without AR) the moments were $4 \mathrm{~N} \cdot \mathrm{m}$. Typically, the resultant moments increased with increasing angle (see Picture, Supplemental Digital Content 3, http:/links.lww.com/ BRS/B169, which shows moment and force curves over cycles) and were greatest in the first cycle of each load increment, where failure most frequently occurred. Lowest maximum moments for creation of an EPJF were $25.0 \mathrm{~N} \cdot \mathrm{m}$ $(\mathrm{FL}), 20.0 \mathrm{~N} \cdot \mathrm{m}(\mathrm{LB})$, and $4.0 \mathrm{~N} \cdot \mathrm{m}(\mathrm{AR})$ (specimen of group $3)$, whereas the highest measured moments at a specimen, where no failure occurred, were $50.0 \mathrm{~N} \cdot \mathrm{m}(\mathrm{FL}), 10.0 \mathrm{~N} \cdot \mathrm{m}$ (LB), and $13.0 \mathrm{~N} \cdot \mathrm{m}(\mathrm{AR})$ (group 4). Deviations between the desired and the actual forces were reached with rather small deviations at the cyclic speed of $2 \mathrm{~Hz}$. In groups 1, 3, 4, and 5 , axial compressive force declined from an offset of $100 \mathrm{~N}$ during the first 20 cycles to less than $50 \mathrm{~N}$ after 40 cycles. In group 2, the actual force was kept within $0 \pm 100 \mathrm{~N}$ (see Picture, Supplemental Digital Content Picture 3, http:// links.lww.com/BRS/B169, which shows moment and force curves over cycles).

\begin{tabular}{|c|c|c|c|c|c|}
\hline & $\begin{array}{c}\text { Group } 1 \\
\text { All Combined }\end{array}$ & $\begin{array}{l}\text { Group } 2 \\
\text { w/o AC }\end{array}$ & $\begin{array}{l}\text { Group } 3 \\
\text { w/o AR }\end{array}$ & $\begin{array}{c}\text { Group } 4 \\
\text { w/o LB }\end{array}$ & $\begin{array}{l}\text { Group } 5 \\
\text { w/o FL }\end{array}$ \\
\hline Crackling noise $\left(\right.$ cycle) ${ }^{*}$ & $70(30-100)$ & $76(50-90)$ & $70(70-100)$ & $81(80-90)$ & - \\
\hline Peak moments FL $(\mathrm{N} \cdot \mathrm{m})^{*}$ & $52.5(40-70)$ & $37.5(30-50)$ & $32.5(20-50)$ & $30.0(20-50)$ & $10.0(0-20)$ \\
\hline Peak moments LB $(\mathrm{N} \cdot \mathrm{m})^{*}$ & $14.0(10-26)$ & $14.0(7-17)$ & $16.5(5-20)$ & $7.0(5-10)$ & $10.0(5-18)$ \\
\hline Peak moments AR $(\mathrm{N} \cdot \mathrm{m})^{*}$ & $14.0(12-16)$ & $12.5(10-15)$ & $4.0(2-5)$ & $12.0(5-15)$ & $13.0(10-18)$ \\
\hline $\begin{array}{l}\text { Sole annulus failure/along } \\
\text { with EPJF }\end{array}$ & $0 / 3$ & $0 / 5$ & $2 / 1$ & $2 / 0$ & $0 / 0$ \\
\hline Large EPJF (caudal/cranial) & $6(5 / 1)$ & $3(2 / 1)$ & $3(2 / 1)$ & $1(1 / 0)$ & 0 \\
\hline Small EPJF & 0 & 3 & 0 & 1 & 0 \\
\hline Risk for EPJF & 6 & 4.5 & 3 & 1.5 & 0 \\
\hline
\end{tabular}




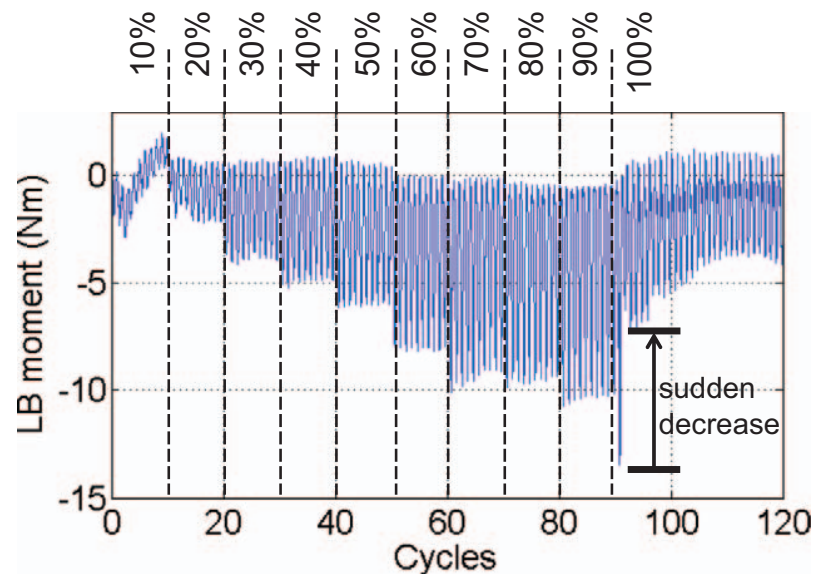

Figure 2. Typical sudden decrease in the magnitude of the measured reaction moment usually after the first cycle of a load increment, indicating failure (shown here at $100 \%$ load). LB indicates lateral bending.

In several cases, crackling noises/visible defects at the posterior part of the disc captured in the video documentation correlated with a decrease in the measured moments (Figure 2). Disc bulging in cases of failure was also evident from video documentation (Figure 3).

In $\mu \mathrm{CT}$ images, the cortical shell of the vertebrae and the trabecular structure could be differentiated. Separation of the discs from the adjacent vertebral bodies was possible, whereas nucleus and annulus could not be distinguished. Density variations in the disc could, however, be recognized. In some cases, bright spots (higher density) and dark spots (lower density) were observed (Figure 4). Endplate defects could easily be detected and appeared as smaller or larger bony parts torn apart from the vertebral body with the crack gap reaching either the annulus or the nucleus (Figures 5 and 6).

MR images had a much higher contrast within the disc but low signal from bony parts. Nucleus and annulus were easily distinguishable and annular lamellae could be visualized. The outer posterior annulus usually had low signal intensity in MR images and presented as an area of absorption equal to the surrounding soft tissue in $\mu \mathrm{CT}$. Annulus failures appeared as dark spots in both MR and $\mu \mathrm{CT}$ images (Figure 7). Usually an annulus failure was seen in the outer part of the posterior annulus. Calcification occurred in 19 discs and appeared as white spots in $\mu \mathrm{CT}$ and dark spots in the respective MR images (Figure 4).

When an EPJF with a crack gap reaching the nucleus occurred, the cartilaginous endplate remained intact so that no nuclear material extruded (Figure 6). In only one case did the cartilaginous endplate brake making nuclear material visible inside the vertebral body; the nuclear material did not further extrude (Figure 6).

\section{DISCUSSION}

In the present study, four motion parameters were systematically analyzed for their influence on the initiation of intervertebral disc damage under a cyclic, complex loading

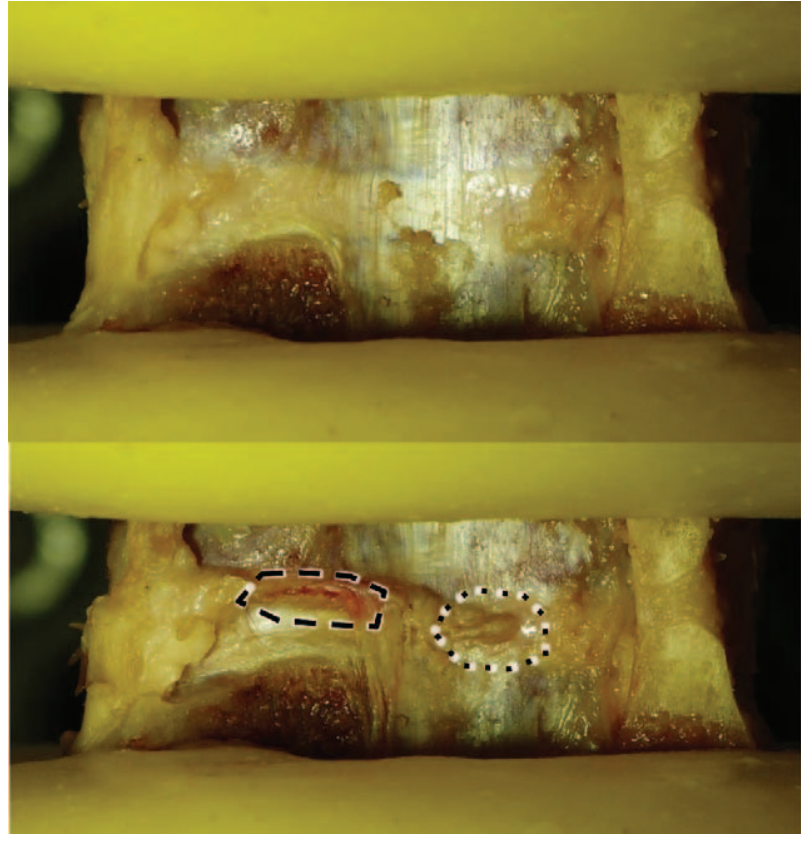

Figure 3. Comparison of the posterior annulus before (top) and after mechanical loading (bottom). Bulging of the disc (dashed line) and endplate junction failure (EPJF) (dotted line) are visible (bottom).

protocol. It was shown that several complex loading protocols can lead to annulus and endplate failures. The combination of FL and LB seems crucial in the generation of failure. A total of 21 specimens (70\%) failed of which the majority was EPJF (76\%), whereas the rest were annulus failures. Herniations, however, did not occur. Because annulus failures occurred only in the posterior outer annulus they were not included in risk calculation.

A limitation of the present study is the usage of ovine specimens, restricting the transferability to human specimens. It was previously shown that ovine lumbar specimens,
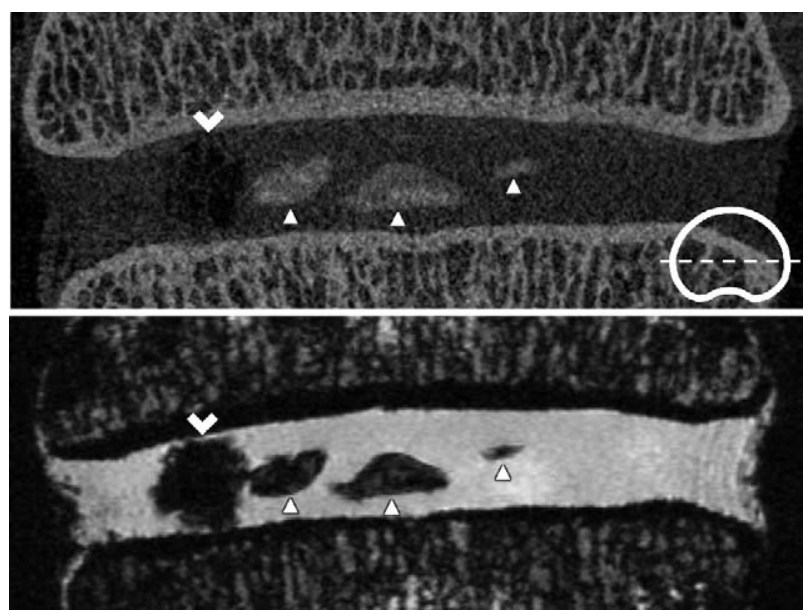

Figure 4. Frontal cross-sections of a tested L3-4 specimen. Calcification (triangles) is seen as bright spots in micro-computed tomography $(\mu \mathrm{CT})$ (top) and dark spots in magnetic resonance imaging (MRI) (bottom). In contrast, gas accumulation (arrow) in the nucleus-annulus transition zone is seen dark in both MRI and $\mu \mathrm{CT}$. 

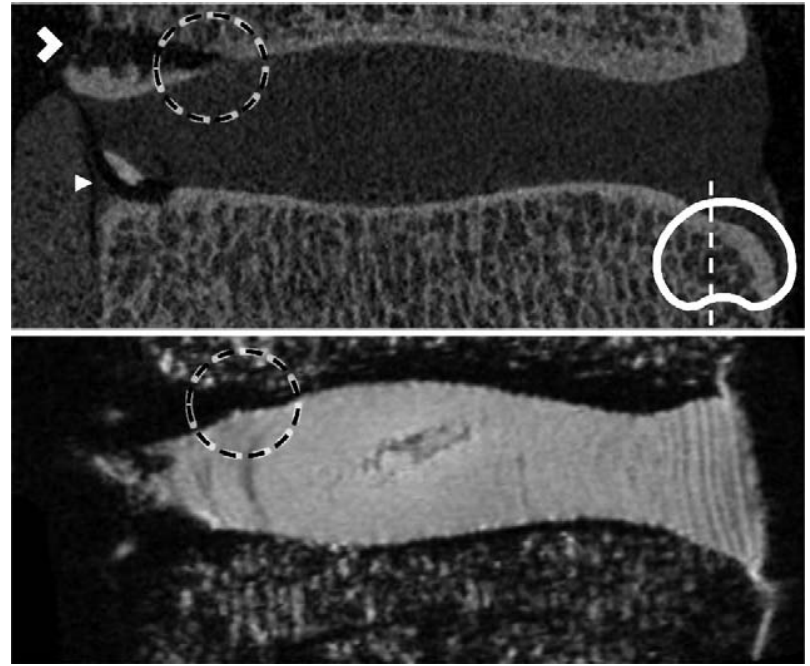

Figure 5. Sagittal cross-sections of a failed L5-6 disc. Micro-computed tomography $(\mu \mathrm{CT})$ (top) reveals a large cranial endplate junction failure (EPJF) (arrow) and a small caudal EPJF (triangle) as an avulsion of the cortical shell. Comparison with the respective magnetic resonance imaging (MRI) (bottom) shows that the large EPJF reaches the inner annulus and that the cartilaginous endplate is intact (circle).

however, have a strong anatomical similarity to human lumbar specimens ${ }^{25}$ and are biomechanically comparable. $^{26,27}$ Furthermore, healthy ovine specimens are available in large numbers. The same damage pattern as in human spinal motion segments could be produced in vitro, as shown by several publications ${ }^{18,20,28}$ and the present study. Thus, ovine specimens cannot replace human specimens, but they seem to be a suitable model for the current scientific approach. Removal of the posterior elements alters the biomechanics of the motion segment and is thus another limitation. This, however, allowed filming of the posterior part of the disc.

As evident from video documentation in addition to MRI, the lesions usually occurred at the lateral left or left paramedian part of the posterior disc. This was expected

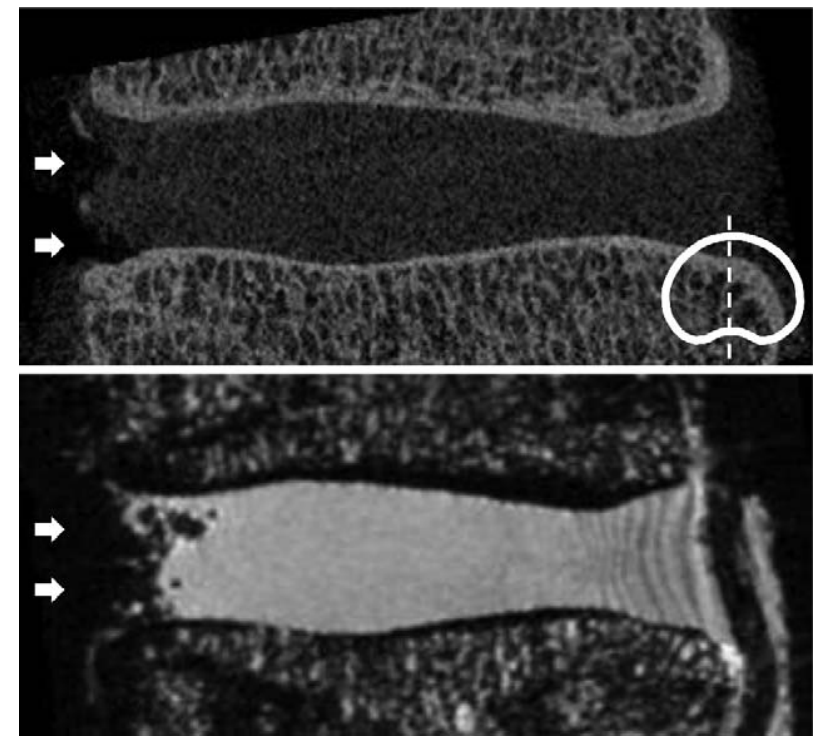

Figure 7. Midsagittal cross-sections of a failed L3-4 disc. Microcomputed tomography $(\mu \mathrm{CT})$ (top) reveals a failure of the outer posterior annulus as dark areas (arrows) indicating no absorption. The same areas in the respective magnetic resonance imaging (MRI) (bottom) have no signal (arrows), which shows that the annulus is torn.

because FL stresses the posterior part and right LB the left part of the disc. The moments observed for FL were much higher than that for LB and AR, even though twice the values of the range of motion recorded under a $3.75 \mathrm{~N} \cdot \mathrm{m}$ moment were used for all axes. Thus, elastic zone stiffness ${ }^{29}$ increased much faster in FL than in LB and AR. Furthermore, only FL moments were dependent on the compressive load. Therefore, the moments in group 2 (w/o AC) were lower than in group 1 . It cannot, however, be explained why FL moments of group 3 and 4 did not reach the moments of group 1. It is assumed that AR and LB have an effect on the resultant FL moment. Even if there was no load applied in one of the four loading directions, the measured moment was unequal to zero, probably because of coupled motions
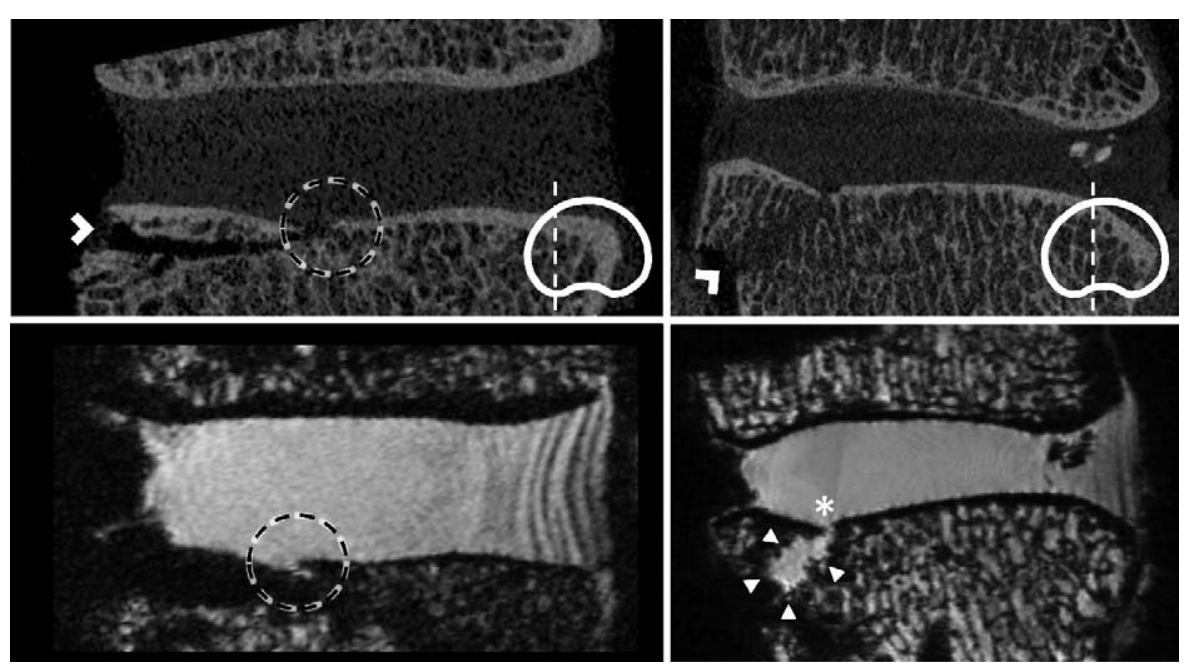

Figure 6. Sagittal cross-sections of a failed L2-3 disc (left) and L5-6 disc (right). Micro-computed tomography $(\mu \mathrm{CT})$ (top) reveals a large caudal endplate junction failure (EPJF) (arrow). Comparison with respective magnetic resonance imaging (MRI) (bottom) shows that the EPJF reaches the nucleus and that the cartilaginous endplate is intact (circle). Rupture of the cartilaginous endplate (asterisk) allows nuclear material to be extruded into the fracture gap (triangles). 
in the FSU. Still, the measured values were smaller than when load was applied in the respective direction. The first cycle of each load step showed a higher reaction moment than the following nine cycles due to relaxation of the soft tissue. This makes the disc vulnerable to traumatic overloads and explains why failure occurred at the first cycle of a specific load increment.

The crackling sound occurring during EPJF maybe related to patient anamnesis statements, which often report a sensation of cracking or popping during injury of the spine.

For the creation of an EPJF, the lowest maximum moments were smaller compared to the highest maximum moments of a specimen where no failure occurred. In general, the resultant moments of each specimen varied significantly within the groups. Therefore, an exact moment to failure cannot be derived from the results. Rather, the angles used for the load protocol seem to play a crucial role.

Using MRI, the detection of EPJF without nuclear extrusion was barely possible due to low signal from bone while $\mu \mathrm{CT}$, with a resolution of $34 \mu \mathrm{m}$ (isometric), revealed such defects easily. However, using standard clinical imaging, visualization of these defects could be problematic. In contrast to our previous study, ${ }^{24}$ visualization of an annular lesion from MRI was unfavorable because the signal from the posterior annulus was low, due to air-tissue interfaces and/or intrinsic low signal from ligaments. Nevertheless, data from video documentation and $\mu \mathrm{CT}$ compensated for this.

Care must be taken in the analysis of calcified discs, which occur in human ${ }^{30}$ but also in sheep ${ }^{31}$ starting from the age of 4 . In MRI, this can be mistakenly identified as the so-called vacuum phenomenon. The existence of gas can be assumed only if CT shows less absorption in an area in which MRI detects low signal. To distinguish between gas, lesion, and calcification, both $\mathrm{MR}$ and $\mu \mathrm{CT}$ images are therefore essential.

The combination of FL, LB, AR, and AC has the highest risk for caudal EPJF. FL without LB and vice versa has the lowest risk for herniation. The absence of AR (group 3) is associated with a lower risk for EPJF compared to the absence of AC (group 2). Thus, AR has a higher effect on the initiation of EPJFs. Because group 4 shows that FL alone rarely produces herniation and LB is not able at all (group
5), FL seems more vulnerable than LB. In summary, the combination of FL and LB seems to be crucial for the initiation of failure and even more if combined with AR and/or AC.

The ratio of EPJF to annulus failure in this sheep study was slightly higher than that observed previously ${ }^{19}$ but still in the same order of magnitude. The percentage of lower EPJFs $(77 \%)$ can be explained by the thinner and thus weaker cranial vertebral endplate ${ }^{32,33}$ which further supports the clinical findings. ${ }^{19}$

In a previous study ${ }^{20}$ using a loading protocol similar to group 1 but with axial loads occasionally exceeding $1 \mathrm{kN}$, EPJFs with herniations were artificially induced. Therefore, it is conceivable that higher compressive load could lead to a rupture of the cartilaginous endplate and/or the remaining posterior annulus. Eventually, nucleus material will be gradually pressed through the fracture gap and result in a herniation (Figure 8). It is also reasonable that with higher $\mathrm{AC}$ the shear forces in the annulus/endplate transition are higher because of the increased pressure of the nucleus, leading to annulus failure in this region. ${ }^{28,34}$ In contrast, larger angles and complex loading with less compression lead mainly to tensile stress on the annulus/endplate region resulting in an avulsion of the endplate as shown in the present study and in a previous study. ${ }^{20}$ Furthermore, it seems that if no compressive load is given using complex loading, as in group 2, the extent of endplate avulsions decreases while additional annulus failures occur. This evidence demonstrates that the type of failure highly depends on how the motion segment is loaded.

The EPJF variety seems to be a subset of what is actually seen in clinical practice where there is an extruded or nearly sequestrated disc herniation just inferior to or superior to the annular/bony margin with migration. The visible annular fibers seem to be intact in these cases. The present study may lead to subtle imaging features closely evaluating the endplate for abnormal findings that might predict future endplate fracture and possible disc herniations through these areas of weakness, much like the current ability to visualize high intensity zones in the outer annulus which could be pain sensitive lesions.

By now, it is known that there is a direct pathway from mechanical loading to herniation. In another indirect
Figure 8. Hypothesis for the process of lumbar disc herniation. A healthy disc (left) is traumatically loaded, initiating an endplate defect that does not affect the cartilaginous endplate (middle). After additional axial compression, the cartilaginous endplate ruptures and eventually nuclear material is pressed out forming a herniation (right).
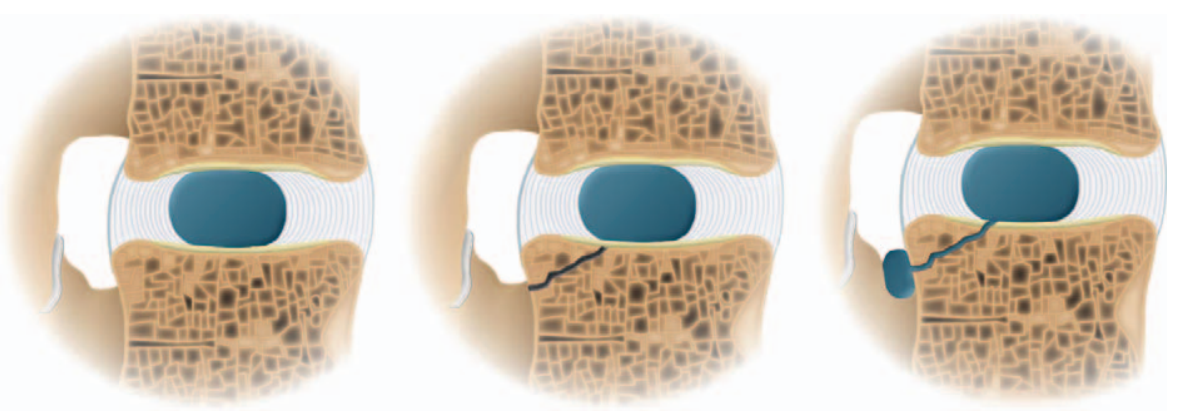
pathway other factors such as genetics in combination with mechanical loading may induce degenerative processes which may finally lead to herniation. ${ }^{35,36}$ It has been shown previously by several in vitro organ culture models applying various loading protocols ${ }^{37-41}$ that mechanical stress may lead to a degenerative cascade, initiating disc degeneration. Our loading protocol may be compared to some of the ones conducted in the studies mentioned above. Thus, in the midto long term the loading presented may induce degenerative processes which may weaken the structure.

\section{CONCLUSION}

The findings of the present study demonstrate that complex overloading of a spine segment inevitably leads to failure. Endplate failures seem to occur more often than annulus failures if physiological compression is used with the combination of at least FL and LB. Herniations are, however, not necessarily the result of such failures. Visualization of endplate (or annulus) failure without herniation by standard CT or MRI might be difficult. Therefore, it is suggested to investigate preventive measures and timely recognition and diagnosis. In addition, further research into the failure mechanism of the endplate and annulus is crucial to fully understand how, where, and why an intervertebral disc fails.

\section{Key Points}

FL and $L B$ are required for the initiation of endplate failure.

Highest risk for spinal motion segment failure is under the combination of $F L, L B, A R$, and $A C$.

Further loading and/or additional $A C$ can lead to delayed herniation.

Chances of recognition and diagnosis of initial failure are small.

Further research into the endplate failure mechanism and its timely recognition is suggested.

\section{Acknowledgment}

The authors thank Anne Subgang for her assistance in scanning the discs, and René Jonas, Christian Liebsch, and Nicholaus Meyers for carefully reading the manuscript.

Supplemental digital content is available for this article. Direct URL citations appearing in the printed text are provided in the HTML and PDF version of this article on the journal's Web site (www.spinejournal.com).

\section{References}

1. Virgin WJ. Experimental investigations into the physical properties of the intervertebral disc. J Bone Joint Surg Br 1951;33:607-11.

2. Hirsch C. The reaction of intervertebral discs to compression forces. J Bone Joint Surg Am 1955;37:1188-96.

3. Brown T, Hansen RJ, Yorra AJ. Some mechanical tests on the lumbosacral spine with particular reference to the intervertebral discs—a preliminary report. J Bone Joint Surg Am 1957;39:1135-64.
4. Hardy WG, Lissner HR, Webster JE, et al. Repeated loading tests of the lumbar spine: a preliminary report. Surg Forum 1958;9: 690-5.

5. Roaf R. A Study of the mechanics of spinal injuries. J Bone Joint Surg Br 1960;42:810-23.

6. Farfan HF, Cossette JW, Robertson GH, et al. The effects of torsion on the lumbar intervertebral joints: the role of torsion in the production of disc degeneration. J Bone Joint Surg Am 1970;52:468-97.

7. Adams MA, Hutton WC. The relevance of torsion to the mechanical derangement of the lumbar spine. Spine 1981;6:241-8.

8. Liu YK, Goel VK, Dejong A, et al. Torsional fatigue of the lumbar intervertebral joints. Spine 1985;10:894-900.

9. Hutton WC, Adams MA. Can the lumbar spine be crushed in heavy lifting? Spine 1982;7:586-90.

10. Adams MA, Hutton WC. Prolapsed intervertebral disc. a hyperflexion injury 1981 Volvo Award in Basic Science. Spine 1982;7:184-91.

11. Adams MA, Hutton WC. The mechanics of prolapsed intervertebral disc. Int Orthop 1982;6:249-53.

12. Adams MA, Hutton WC. The effect of fatigue on the lumbar intervertebral disc. J Bone Joint Surg Br 1983;65:199-203.

13. Adams MA, Hutton WC. Gradual disc prolapse. Spine 1985;10: 524-31.

14. Drake JDM, Aultman CD, McGill SM, et al. The influence of static axial torque in combined loading on intervertebral joint failure mechanics using a porcine model. Clin Biomech (Bristol, Avon) 2005;20:1038-45.

15. Marshall LW, McGill SM. The role of axial torque in disc herniation. Clin Biomech (Bristol, Avon) 2010;25:6-9.

16. Veres SP, Robertson PA, Broom ND. The influence of torsion on disc herniation when combined with flexion. Eur Spine J 2010;19:1468-78.

17. Schmidt H, Kettler A, Heuer F, et al. Intradiscal pressure, shear strain, and fiber strain in the intervertebral disc under combined loading. Spine (Phila Pa 1976) 2007;32:748-55.

18. Veres SP, Robertson PA, Broom ND. The morphology of acute disc herniation: a clinically relevant model defining the role of flexion. Spine (Phila Pa 1976) 2009;34:2288-96.

19. Rajasekaran S, Bajaj N, Tubaki V, et al. ISSLS prize winner: the anatomy of failure in lumbar disc herniation: an in vivo, multimodal, prospective study of 181 subjects. Spine (Phila Pa 1976) 2013;38:1491-500.

20. Wilke H-J, Kienle A, Maile S, et al. A new dynamic six degrees of freedom disc-loading simulator allows to provoke disc damage and herniation. Eur Spine J 2016;25:1363-72.

21. Reitmaier S, Schmidt H, Ihler R, et al. Preliminary investigations on intradiscal pressures during daily activities: an in vivo study using the merino sheep. PLoS One 2013;8:e69610.

22. Reitmaier S, Volkheimer D, Berger-Roscher N, et al. Increase or decrease in stability after nucleotomy? Conflicting in vitro and in vivo results in the sheep model. $J$ R Soc Interface 2014;11: 20140650.

23. Consmuller T, Rohlmann A, Weinland D, et al. Velocity of lordosis angle during spinal flexion and extension. Plos One 2012;7: e50135.

24. Berger-Roscher N, Galbusera F, Rasche V, et al. Intervertebral disc lesions: visualisation with ultra-high field MRI at 11.7 T. Eur Spine J 2015;24:2488-95.

25. Wilke HJ, Kettler A, Wenger KH, et al. Anatomy of the sheep spine and its comparison to the human spine. Anat Rec 1997;247:542-55.

26. Schmidt H, Reitmaier S. Is the ovine intervertebral disc a small human one? A finite element model study. J Mech Behav Biomed Mater 2013;17:229-41.

27. Wilke HJ, Kettler A, Claes LE. Are sheep spines a valid biomechanical model for human spines? Spine (Phila Pa 1976) 1997;22:2365-74.

28. Wade KR, Robertson PA, Thambyah A, et al. Surprise loading in flexion increases the risk of disc herniation due to annulus-endplate junction failure: a mechanical and microstructural investigation. Spine (Phila Pa 1976) 2015;40:891-901. 
29. Wilke HJ, Wenger K, Claes L. Testing criteria for spinal implants: recommendations for the standardization of in vitro stability testing of spinal implants. Eur Spine J 1998;7:148-54.

30. Chanchairujira K, Chung CB, Kim JY, et al. Intervertebral disk calcification of the spine in an elderly population: radiographic prevalence, location, and distribution and correlation with spinal degeneration. Radiology 2004;230: 499-503.

31. Melrose J, Burkhardt D, Taylor TK, et al. Calcification in the ovine intervertebral disc: a model of hydroxyapatite deposition disease. Eur Spine J 2009;18:479-89.

32. Grant JP, Oxland TR, Dvorak MF. Mapping the structural properties of the lumbosacral vertebral endplates. Spine (Phila Pa 1976) 2001;26:889-96.

33. Zhao FD, Pollintine P, Hole BD, et al. Vertebral fractures usually affect the cranial endplate because it is thinner and supported by less-dense trabecular bone. Bone 2009;44:372-9.

34. Wade KR, Robertson PA, Thambyah A, et al. How healthy discs herniate: a biomechanical and microstructural study investigating the combined effects of compression rate and flexion. Spine (Phila Pa 1976) 2014;39:1018-28.
35. Adams MA, Roughley PJ. What is intervertebral disc degeneration, and what causes it? Spine (Phila Pa 1976) 2006;31:2151-61.

36. Battie MC, Videman T, Kaprio J, et al. The Twin Spine Study: contributions to a changing view of disc degeneration. Spine J 2009;9:47-59.

37. Alkhatib B, Rosenzweig DH, Krock E, et al. Acute mechanical injury of the human intervertebral disc: link to degeneration and pain. Eur Cell Mater 2014;28:98-110.

38. Chan SC, Walser J, Ferguson SJ, et al. Duration-dependent influence of dynamic torsion on the intervertebral disc: an intact disc organ culture study. Eur Spine J 2015;24:2402-10.

39. Chan SC, Walser J, Kappeli P, et al. Region specific response of intervertebral disc cells to complex dynamic loading: an organ culture study using a dynamic torsion-compression bioreactor. Plos One 2013;8:e72489.

40. Dudli S, Haschtmann D, Ferguson SJ. Fracture of the vertebral endplates, but not equienergetic impact load, promotes disc degeneration in vitro. J Orthop Res 2012;30:809-16.

41. Walter BA, Korecki CL, Purmessur D, et al. Complex loading affects intervertebral disc mechanics and biology. Osteoarthritis Cartilage 2011;19:1011-8. 